\title{
Ação anti-helmíntica da Morinda citrifolia (noni) sobre Heterakis gallinarum
}

\author{
Anthelmintic action of Morinda citrifolia (noni) on \\ Heterakis gallinarum
}

\author{
Danilo Rodrigues Barros Brito ${ }^{1 *}$; Rozeverter Moreno Fernandes ${ }^{2}$
}

\section{Resumo}

\begin{abstract}
A ação anti-helmíntica da Morinda citrifolia (noni) sobre Heterakis gallinarum foi avaliada em galinhas poedeiras naturalmente infectadas. A atividade anti-helmíntica in vitro foi determinada em helmintos adultos colocados em placas de petri descartáveis, contendo solução Tyrode pré-aquecida, nas quais foi adicionado o extrato aquoso ou etanólico e mantidas em BOD a uma temperatura de $37^{\circ} \mathrm{C}( \pm 1)$. Os extratos aquoso e etanólico foram usados nas seguintes concentrações: 1,$69 ; 3,37 ; 6,74 ; 13,48$ e 26,96 mg.mL ${ }^{-1}$ e 4,$17 ; 8,34 ; 16,68 ; 33,36$ e 66,72 mg.mL ${ }^{-1}$, respectivamente. Como controle positivo usouse uma solução de citrato de piperazina tetrahidratada na concentração de $50 \mathrm{mg} \cdot \mathrm{mL}^{-1}$. A atividade anti-helmíntica in vivo foi determinada em aves, administrando-se os extratos aquoso ou etanólico (10 $\mathrm{mL} / \mathrm{Kg} / \mathrm{PV}$ ) durante três dias consecutivos. As fezes dos animais foram coletadas durante quatro dias, em seguida foram lavadas em água corrente e peneiradas. No quinto dia após início do tratamento, as aves foram abatidas e necropsiadas, para contagem e identificação dos helmintos remanescentes. Os dados obtidos foram analisados estatisticamente utilizando o teste de Student-Newman-Keuls. No teste in vivo, não houve diferença significativa entre o extrato aquoso (concentração de $10 \%$ ) e o grupo controle (água) ( $>>0,05)$ na eliminação de $H$. gallinarum. O extrato etanólico apresentou um percentual de eliminação de 20,35\%, diferindo estatisticamente do grupo controle $(\mathrm{p}<0,05)$. Na concentração 26,96 $\mathrm{mg} . \mathrm{mL}^{-1}$, para o teste in vitro, o extrato aquoso causou mortalidade de $100 \%$, semelhante ao obtido pela piperazina $(100 \%)$, diferindo estatisticamente do controle negativo $(\mathrm{p}<0,05)$. Nas concentrações 33,36 e $66,72 \mathrm{mg} \cdot \mathrm{mL}^{-1}$, o extrato etanólico também apresentou percentagem de mortalidade de $100 \%$, havendo diferença estatisticamente significativa do controle negativo $(\mathrm{P}<0,05)$. Conclui-se que a atividade antihelmíntica do fruto do noni apresentou resultados promissores no teste in vitro, havendo necessidade de estudos com maiores concentrações no teste in vivo.
\end{abstract}

Palavras-chave: Plantas medicinais, helmintologia, galinha

\section{Abstract}

The anthelmintic effect of Morinda citrifolia (noni) on Heterakis gallinarum was evaluated in chicken naturally infected. The anthelmintic activity in vitro was determined in adults helminthes in disposable petri dishes, containing Tyrode solution, pre warmed in which aqueous or ethanolic extracts were added. The material was maintained in a BOD at $37^{\circ} \mathrm{C}( \pm 1)$. The aqueous and ethanolic extracts presented the following concentrations: $1.69 ; 3.37 ; 6.74 ; 13.48$ e $26.96 \mathrm{mg} \cdot \mathrm{mL}^{-1}$ and $4.17 ; 8.34 ; 16.68 ; 33.36$ e 66.72 $\mathrm{mg} \cdot \mathrm{mL}^{-1}$, respectively. It was used as positive control, a solution of tetrahidrate citrate of piperazin in the concentration of $50 \mathrm{mg} / \mathrm{mL}$. The anthelmintic activity in vivo was determined by the administration of aqueous or ethanolic extracts $(10 \mathrm{~mL} / \mathrm{Kg} / \mathrm{PV})$ during three consecutive days. The feces were collected during four days in each group, washed in water and sifted. In the fifth day post-treatment, the chickens

1 Prof. do Instituto Federal do Maranhão, IFMA, Campus São Luís-Maracanã, São Luís, MA. E-mail: danilobrito@ifma.edu.br

2 Prof. da Universidade Federal do Piauí, UFPI, Teresina, PI. E-mail: zmoreno48@gmail.com

Autor para correspondência

Recebido para publicação 21/05/12 Aprovado em 05/05/13 
were slaughtered and necropsy was performed in order to count and identify remaining helminthes. The data were analyzed by the Student-Newman-Keuls test. In the in vivo test there was no significant difference between the aqueous extract and the control group (water) ( $>0.05)$ in the elimination of $H$. gallinarum. The ethanolic extract presented an elimination of $20.35 \%$, differing statistically from the control group $(\mathrm{p}<0.05)$. In the concentration of $26.96 \mathrm{mg} \cdot \mathrm{mL}^{-1}$, for the in vitro test, the aqueous extract presented a mortality of $100 \%$, the same obtained by piperazin $(100 \%)$, differing statistically from the negative control $(\mathrm{p}<0.05)$. In the concentrations of 33.36 and $66.72 \mathrm{mg} \cdot \mathrm{mL}^{-1}$, the ethanolic extract also presented a mortality of $100 \%$, there was a significant statistical difference from the negative control $(\mathrm{p}<0,05)$. Noni fruit extracts manifested high anthelmintic activity in vitro, however, more studies are necessary with higher concentrations in the in vivo test.

Key words: Medicinal plants, helminthology, chicken

\section{Introdução}

$\mathrm{Na}$ área farmacêutica, as plantas e os extratos vegetais foram e continuam sendo de grande relevância, tendo em vista a utilização das substâncias ativas como protótipos para o desenvolvimento de novas drogas e como fonte de matérias-primas, tanto para a obtenção de fármacos (que são as substâncias ativas isoladas), como para a obtenção de adjuvantes (produtos utilizados na formulação de medicamentos) ou, ainda, de produtos elaborados exclusivamente à base de extratos vegetais: os fitoterápicos (SCHENKEL; GOSMANN; PETROVICK, 2001).

Considerando a produção alternativa de frangos (frango colonial), que visa reproduzir ao máximo as condições naturais de vida da ave, busca-se produzir alimentos saudáveis, de elevado valor nutricional e isentos de contaminantes, preservando a biodiversidade em que se insere o sistema produtivo. Para tanto é necessário adotar práticas de produção menos agressivas e que aperfeiçoem o uso de recursos naturais, tendo por objetivo a autosustentação (FIGUEIREDO et al., 2001).

Diversas plantas têm sido estudadas com a finalidade de substituição da utilização de produtos químicos por compostos naturais que poderão ter eficácia semelhante, melhorando a questão ambiental e a economicidade. Dentre essas plantas, a Morinda citrifolia, conhecida popularmente como noni, muito disseminada na região Nordeste do Brasil, tem mostrado ser uma planta promissora para ser usada na eliminação de helmintos parasitas de aves.
Heterakis gallinarum é um nematoide pequeno, mede entre 0,4 e 1,5 centímetros. Os hospedeiros intermediários são oligoquetas dos gêneros Lumbricus, Allolobophora e Eisenia, e alguns insetos, que ingerem ovos do parasita. As galinhas se infectam quando ingerem estes hospedeiros intermediários, que contenham a forma infectante localizada nos tecidos. Parasitam o ceco e os adultos são patogênicos, causando tiflite, diarréia e perda de peso. Os ovos de H. gallinarum são semelhantes aos de Ascaridia galli (FREITAS, 1977).

Em estudos realizados com galinhas soltas no município de Seropédica, Rio de Janeiro, Carneiro (2001) encontrou doze espécies de helmintos, sendo sete nematoides e cinco cestódeos. As espécies que apresentaram os maiores graus de dominância foram $H$. gallinarum e Capillaria sp., sendo estas duas espécies classificadas como centrais. Menezes et al. (2001) realizaram uma pesquisa sobre as helmintoses que ocorrem em galinhasd'angola criadas extensivamente no estado do Rio de Janeiro, e foi observada uma prevalência de $H$. gallinarum de $100 \%$. Fernandes (1998) estudando a fauna de helmintos em 70 frangos de corte criados semi-extensivamente, verificou que $94,3 \%$ eram positivos para $\mathrm{A}$. galli e $100 \%$ para $\mathrm{H}$. gallinarum. $\mathrm{O}$ mesmo autor também observou a localização desses helmintos, sendo que $97,7 \%$ de $A$. galli estavam presentes no intestino delgado e $2,3 \%$ no intestino grosso, enquanto $99,2 \%$ de $\mathrm{H}$. gallinarum foram observados no ceco e $0,8 \%$ no cólon.

Segundo Bowman et al. (2006), H. gallinarum serve como hospedeiro paratênico do Histomonas 
meleagridis. Este protozoário ao ser ingerido pelo nematoide vai se localizar dentro do ovo deste parasito. Os ovos contendo $H$. meleagridis são liberados no ambiente e são ingeridos pelas aves. No intestino destas ocorre liberação da larva de $H$. gallinarum juntamente com o protozoário que irá para o ceco onde se multiplicará, podendo causar processo inflamatório severo no fígado e no ceco, com presença de pequenas áreas claras cercadas de eosinófilos.

Brito et al. (2009) avaliando a atividade antihelmíntica dos extratos aquoso e etanólico do fruto da Morinda citrifolia sobre Ascaridia galli, observaram nos testes in vitro, que o extrato aquoso na concentração de $26,96 \mathrm{mg} \cdot \mathrm{mL}^{-1}$ causou mortalidade de $50 \%$ e o extrato etanólico na concentração de $66,72 \mathrm{mg} \cdot \mathrm{mL}^{-1}, 76,67 \%$ após 96 horas de tratamento. Fernandes (2008) estudando a atividade anti-helmíntica in vivo de plantas sobre H. gallinarum, observou que o extrato aquoso de Simarouba versicolor apresentou percentual de eliminação de $20,22 \%$ e o extrato etanólico de 29,15\%. Já o extrato aquoso da Annona squamosa apresentou percentual de eliminação de 20,60\%.

Estudos sobre o efeito anti-helmíntico da folha do noni (M. citrifolia) sobre A. galli, demonstraram que o extrato aquoso da folha do noni, no teste in vitro e na concentração de 13,92 mg.mL $\mathrm{m}^{-1}$, causou mortalidade de $96,67 \%$ após 96 horas de tratamento, assemelhando-se ao controle positivo (piperazina a $50 \mathrm{mg} \cdot \mathrm{mL}^{-1}$ ) que causou $100 \%$ de mortalidade (BRITO et al., 2011). Fernandes et al. (2004) pesquisando a atividade anti-helmíntica das plantas Allium sativum, Punica granatum, Tynnanthus fasciculatus e Cocos nucifera sobre o $\mathrm{H}$. gallinarum em teste in vivo com frangos infectados naturalmente, constataram que o extrato aquoso e suco por gavage dessas plantas incorporadas à ração nas doses de 2,3 e $10 \mathrm{~g} \mathrm{~kg}^{-1}$ dia $^{-1}$, durante três dias consecutivos, não apresentaram atividade significativa $(\mathrm{P}<0,05)$ sobre este helminto.

Matos (2000) afirma que a avaliação de novas drogas vegetais através da pesquisa é o caminho para que se possa fazer o correto aproveitamento das plantas medicinais e seus derivados aplicados a fitoterapia. Devido à alta prevalência de H. gallinarum em aves e sua importância na sanidade e produção das mesmas e dentro da perspectiva da criação orgânica de frangos, a utilização de plantas medicinais aparece como uma alternativa no controle das helmintíases aviárias. Na criação de frangos coloniais em sistema de produção orgânico não é permitido o uso de antihelmínticos sintéticos (produtos comerciais), como tentativa de suprir esta demanda, este trabalho teve como objetivo avaliar a ação anti-helmíntica da $M$. citrifolia sobre $H$. gallinarum proveniente de aves naturalmente infectadas.

\section{Material e Métodos}

Fonte vegetal: Os frutos da planta $M$. citrifolia foram coletados no município de Altos, Estado do Piauí, no mês de julho (período seco), à latitude de $05^{\circ} 02^{\prime} 20^{\prime}$ ' S, longitude de $42^{\circ} 27^{\prime} 39^{\prime \prime} \mathrm{O}$ e a 187 $\mathrm{m}$ de altitude, a $42 \mathrm{~km}$ ao nordeste de Teresina. $\mathrm{O}$ solo local é Podzólico Vermelho Amarelo Distrófico com baixa fertilidade natural (P $5 \mathrm{mg} / \mathrm{dm}^{3}, \mathrm{~K} 14$ $\mathrm{mg} / \mathrm{dm}^{3}$, Al $6 \mathrm{mmol} / \mathrm{dm}^{3}$, e $\mathrm{Ca}+\mathrm{Mg} 8 \mathrm{mmol} / \mathrm{dm}^{3}$ ) e alta acidez $(\mathrm{pH}-5,3)$. A precipitação média anual do município é em torno de $1.297 \mathrm{~mm}$, sendo que cerca de $90 \%$ das chuvas concentram-se no período de novembro a maio. A temperatura média anual está em torno de $25^{\circ} \mathrm{C}$ e a umidade relativa de $67 \%$. A identificação botânica foi realizada no Núcleo de Referência em Ciências Ambientais do Trópico Ecotonal do Nordeste - TROPEN, Teresina-PI, sendo a exsicata depositada sob o número 21.644 no herbário Graziela Barroso.

Os frutos foram picados, dessecados em estufa de circulação forçada de ar, durante oito dias, a uma temperatura máxima de $45^{\circ} \mathrm{C}( \pm 1)$. Após essa etapa, o material foi triturado em moinho, tipo willis, obtendo-se um pó que foi acondicionado em um frasco de vidro âmbar hermeticamente fechado e identificado, onde permaneceu até o momento do preparo dos extratos. 
Preparação dos Extratos: O extrato etanólico foi obtido através de maceração a frio, após quatro extrações sucessivas, sendo filtrado usandose papel de filtro Whatman $n^{\circ} 1$. Em seguida, concentrado em evaporador rotativo sob pressão reduzida, à temperatura entre 42 e $45^{\circ} \mathrm{C}$ e, então, liofilizado. Utilizou-se em média 1,3 L de etanol para cada extração realizada. $O$ extrato aquoso foi obtido através de cocção, ou seja, $50 \mathrm{~g}$ de matéria vegetal (pó) para $500 \mathrm{~mL}$ de água destilada e, em seguida, deixou-se ferver por dois minutos e, após o resfriamento à temperatura ambiente, a solução obtida foi filtrada, obtendo-se então uma solução a $10 \%$.

Foi retirada uma alíquota de $1 \mathrm{~mL}$ de cada extrato, colocada em estufa de circulação forçada de ar, a uma temperatura de $45^{\circ} \mathrm{C}( \pm 1)$, até a obtenção de um peso constante, procedimento realizado em triplicata. A massa média obtida referente a $1 \mathrm{~mL}$ foi relacionada ao respectivo volume total, obtendo-se então a massa total em mg.mL $\mathrm{mL}^{-1}$. Esse procedimento foi realizado para o extrato aquoso com o objetivo de determinar o peso seco e, para o extrato etanólico, para avaliar o rendimento aproximado após a evaporação do etanol.

Manutenção das aves: Durante o experimento, foram adquiridas em granjas da zona rural, da cidade de Teresina-PI, galinhas poedeiras em fase de descarte e que não recebiam vermífugo há pelo menos três meses. Foram colocadas em um galpão composto por uma área coberta, com bebedouros e comedouros, além da cama de maravalha. Os animais tiveram acesso a uma área descoberta e de chão batido, capinada, evitando-se que os animais tivessem acesso a outras plantas. Para determinar o grau de infecção do plantel, foram realizados exames de fezes, pela técnica Willis modificada (WILLIS APUD UENO; GONÇALVES, 1994).

Atividade anti-helmíntica in vitro: Foi determinada em helmintos adultos, machos e fêmeas de H. gallinarum, coletados nos cecos de aves necropsiadas. Os parasitos foram lavados em solução salina $(\mathrm{NaCl}$ 0,9\%), e aqueles considerados ativos foram transferidos para placas de Petri descartáveis (90x15mm), contendo solução Tyrode (KALEYSA RAJ, 1975) pré-aquecida $\left(37^{\circ} \mathrm{C}\right)$, totalizando 20 nematoides por placa. A seguir, foram adicionados os extratos nas seguintes concentrações: 1,69; 3,37; 6,$74 ; 13,48$ e $26,96 \mathrm{mg} \cdot \mathrm{mL}^{-1}$ do extrato aquoso; e 4,$17 ; 8,34 ; 16,68 ; 33,36$ e $66,72 \mathrm{mg} \cdot \mathrm{mL}^{-1}$ do extrato etanólico. Os nematoides foram mantidos em estufa $\mathrm{BOD}$, à temperatura de $37^{\circ} \mathrm{C}( \pm 1)$, e examinados 6, 24 e 48 horas após o tratamento. Os nematoides imóveis, mesmo após breve pressão com estilete, foram considerados mortos (SHILASKAR; PARASAR, 1985). O experimento foi repetido 3 vezes para cada dose, e a porcentagem de parasitas mortos em cada grupo foi calculada. Esse teste foi acompanhado de dois controles negativos constituídos por água e diluente (Tween 80 a 17,5\% acrescido de DMSO a 12,5\%) e um controle positivo com a droga referência, citrato de piperazina (50 $\mathrm{mg} \cdot \mathrm{mL}^{-1}$ ), administrada de acordo com o fabricante (Proverm Tortuga).

Atividade anti-helmíntica in vivo: Os grupos $(n=6)$ compostos de aves com peso médio de $1,5 \mathrm{~kg}$, foram constituídos por controle positivo (piperazina, $50 \mathrm{mg} \cdot \mathrm{mL}^{-1}$ ), controles negativos (água e diluente idêntico ao teste in vitro) e grupos tratados com os extratos aquoso e etanólico de M. citrifolia, nas doses de 50,1 mg. $\mathrm{mL}^{-1}$ e 24,6 $\mathrm{mg} \cdot \mathrm{mL}^{-1}$, respectivamente. . Os extratos aquoso e etanólico foram administrados, durante três dias consecutivos, no volume de $10 \mathrm{~mL} / \mathrm{kg}$, utilizandose uma sonda intragástrica (FERNANDES et al., 2005). Diagnosticada a infecção por exame de fezes, as aves foram transferidas para gaiolas individuais galvanizadas, com fundo removível, onde passaram por um período de adaptação de 72 horas, recebendo diariamente $50 \mathrm{~g}$ de ração $(16 \%$ de $\mathrm{PB}$ ) e água ad libitum. Antes do início dos testes, as aves foram submetidas a um período de jejum de 6 horas, tendo disponível água à vontade. Em todos os grupos as fezes foram coletadas durante quatro dias. Em seguida, foram levadas ao 
laboratório, lavadas em água corrente e peneiradas em tamis USBS-50, abertura 0,297 mm e tyler 48 sob outro tamis de malha USBS-40, abertura 0,42 $\mathrm{mm}$ e tyler 35 colocado no fundo da pia, de modo a reter o resíduo do primeiro tamis. Posteriormente, foram acondicionadas em frascos contendo uma solução de Ácido Acético, Formol e Álcool (AFA) a quente (AMATO; BOEGER; AMATO, 1991) para conservação, a fim de preservar os helmintos eliminados para posterior contagem. No quinto dia de tratamento, as aves foram sacrificadas e necropsiadas de acordo com a Resolução n 714 de 20/06/2002 do Conselho Federal de Medicina Veterinária. Durante a necropsia parasitológica a mucosa do trato gastrintestinal foi raspada e o conteúdo colocado em frascos contendo AFA quente para posterior contagem e identificação dos helmintos remanescentes. O efeito do tratamento foi avaliado pelo método crítico controlado (STEWARD, 1955), adaptado ao presente modelo experimental e expresso em percentual médio de eliminação de $H$. gallinarum, conforme a fórmula abaixo:

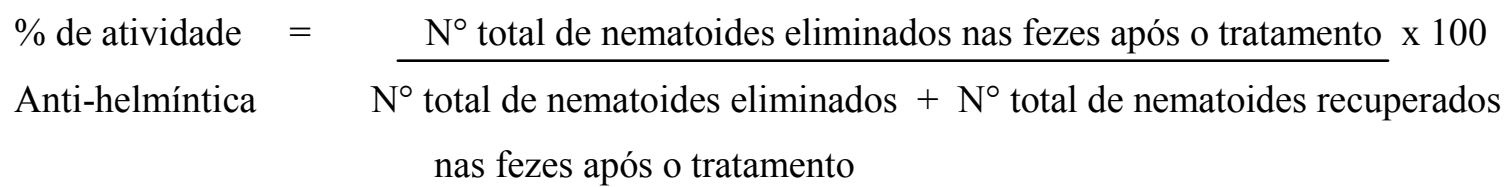

Cálculo semelhante foi aplicado aos grupos controles negativos.

Análise estatística: Os dados obtidos foram analisados estatisticamente, utilizando-se o teste Student-Newman-Keuls, com auxílio do programa InStat (Graphpad Instat: GraphPad Software Oberlin, San Diego - CA, USA). O nível de significância adotado foi $\mathrm{p}<0,05$ (PIMENTELGOMES, 1987).

\section{Resultados e Discussão}

$\mathrm{O} \mathrm{pH}$ do extrato etanólico do fruto do noni foi de 4,40 e, do extrato aquoso, de 3,86. O peso seco do extrato aquoso do fruto do noni foi de $50,1 \mathrm{mg} \cdot \mathrm{mL}^{-1}$ e, do extrato etanólico, de 24,6 mg. $\mathrm{mL}^{-1}$.

A percentagem média de mortalidade nos testes in vitro, usando-se diferentes concentrações do extrato aquoso do fruto de $M$. citrifolia, no intervalo de 48 horas de exposição do parasito ao extrato, está disposta na Tabela 1 .

Considerando as duas maiores concentrações $\left(13,48\right.$ e 26,96 mg.mL $\left.\mathrm{mL}^{-1}\right)$, após seis horas de exposição do $H$. gallinarum, o extrato aquoso do fruto da $M$. citrifolia apresentou percentual de mortalidade superior ao controle positivo (piperazina), diferindo estatisticamente. Após 24h nas concentrações 13,48 e $26,96 \mathrm{mg} \cdot \mathrm{mL}^{-1}$, o percentual de mortalidade não foi significativamente superior ao controle positivo. Com 48 horas de tratamento, o extrato aquoso do fruto da $M$. citrifolia, levando em consideração as duas maiores concentrações (13,48 e 26,96 $\mathrm{mg} \cdot \mathrm{mL}^{-1}$ ), apresentou a mesma eficácia quando comparado ao controle positivo, não diferindo estatisticamente (100\% para ambos tratamentos). Porém quando comparado com controle negativo (28,67\%), houve diferença estatística. 
Tabela 1. Percentual (média \pm desvio padrão) de mortalidade de Heterakis gallinarum no teste in vitro com extrato aquoso do fruto de Morinda citrifolia, ao longo de 48 horas de incubação.

\begin{tabular}{ccccc}
\hline & $\begin{array}{c}\text { Concentração } \\
\left(\mathrm{mg} \cdot \mathrm{mL}^{-1}\right)\end{array}$ & 6 & \multicolumn{2}{c}{ Tempo (horas)/Taxa de Mortalidade (\%) } \\
& 1,69 & $0^{\mathrm{a}}$ & $40 \pm 5^{\mathrm{b}}$ & 48 \\
\hline Extrato & 3,37 & $11,67 \pm 7,64^{\mathrm{a}}$ & $55 \pm 5^{\mathrm{b}}$ & $86,67 \pm 7,64^{\mathrm{b}}$ \\
Aquoso do & 6,74 & $28,33 \pm 2,89^{\mathrm{a}}$ & $66,67 \pm 12,59^{\mathrm{b}}$ & $91,67 \pm 7,64^{\mathrm{c}}$ \\
Noni & 13,48 & $51,67 \pm 10,41^{\mathrm{b}}$ & $86,67 \pm 2,89^{\mathrm{c}}$ & $100 \pm 0^{\mathrm{c}}$ \\
& 26,96 & $88,33 \pm 5,77^{\mathrm{b}}$ & $100 \pm 0^{\mathrm{c}}$ & $100 \pm 0^{\mathrm{c}}$ \\
Piperazina & 50 & $25 \pm 13,23^{\mathrm{a}}$ & $83,33 \pm 12,58^{\mathrm{c}}$ & $100 \pm 0^{\mathrm{c}}$ \\
& & $0^{\mathrm{a}}$ & $4 \pm 1,73^{\mathrm{a}}$ & $28,67 \pm 7,64^{\mathrm{a}}$ \\
\hline Água & & &
\end{tabular}

Médias com letras iguais, na coluna, não diferem entre si pelo teste de Student-Newman-Keuls $(\mathrm{P}>0,05)$.

Fonte: Elaboração dos autores.

Brito et al. (2009), estudando a eficiência da M. citrifolia sobre $A$. galli, encontraram menor eficácia. Observaram percentual de mortalidade inferior a $5 \%$ após $48 \mathrm{~h}$ de tratamento. A maior concentração utilizada foi também de 26,96 $\mathrm{mg} \cdot \mathrm{mL}^{-1}$, demonstrando possivelmente uma maior sensibilidade por parte de H. gallinarum ao extrato aquoso do fruto da $M$. citrifolia no teste in vitro, bem como para piperazina na concentração de 50 $\mathrm{mg} \cdot \mathrm{mL}^{-1}$.

A ação do extrato etanólico do fruto $M$. citrifolia sobre $H$. gallinarum apresentou resultados semelhantes ao extrato aquoso, conforme mostra a Tabela 2. Após $48 \mathrm{~h}$ de tratamento, levando em consideração as duas maiores concentrações $(33,36$ e $\left.66,72 \mathrm{mg} \cdot \mathrm{mL}^{-1}\right)$, o extrato etanólico apresentou o mesmo percentual de mortalidade do controle positivo (piperazina), sendo o mesmo fato observado com o extrato aquoso. As concentrações 8,34; 16,68; 33,36 e 66,72 mg.mL $\mathrm{m}^{-1}$, após 24 e 48h de tratamento, apresentaram diferença significativa $(\mathrm{P}<0,05)$, considerando o extrato etanólico do fruto do noni, controle positivo (piperazina) e o controle negativo (diluente). Nas três maiores concentrações $(16,68$; 33,36 e $66,72 \mathrm{mg} \cdot \mathrm{mL}^{-1)}$, após $48 \mathrm{~h}$ de tratamento, não houve diferença significativa entre extrato etanólico e o controle positivo. Considerando todos os tempos de exposição $(6,24$ e $48 \mathrm{~h})$ e as três maiores concentrações $\left(16,68 ; 33,36\right.$ e $\left.66,72 \mathrm{mg} \cdot \mathrm{mL}^{-1}\right)$, o extrato etanólico do noni diferiu estatisticamente do controle negativo.

Tabela 2. Percentual (média \pm desvio padrão) de mortalidade de Heterakis gallinarum no teste in vitro com extrato etanólico do fruto de Morinda citrifolia, ao longo de 48 horas de incubação.

\begin{tabular}{ccccc}
\hline & $\begin{array}{c}\text { Concentração } \\
\left(\mathrm{mg} \cdot \mathrm{mL}^{-1}\right)\end{array}$ & 6 & \multicolumn{3}{c}{ Tempo (horas)/Taxa de Mortalidade (\%) } \\
& 4,17 & $0^{\mathrm{a}}$ & $10 \pm 5^{\mathrm{a}}$ & 48 \\
\hline Extrato & 8,34 & $0^{\mathrm{a}}$ & $35 \pm 5^{\mathrm{b}}$ & $45 \pm 5^{\mathrm{b}}$ \\
Etanólico do & 16,68 & $10 \pm 10^{\mathrm{ab}}$ & $48,33 \pm 2,89^{\mathrm{b}}$ & $93,33 \pm 2,89^{\mathrm{c}}$ \\
noni & 33,36 & $23,33 \pm 7,64^{\mathrm{b}}$ & $80 \pm 10^{\mathrm{c}}$ & $100 \pm 0^{\mathrm{d}}$ \\
& 66,72 & $73,33 \pm 7,64^{\mathrm{c}}$ & $100 \pm 0^{\mathrm{c}}$ & $100 \pm 0^{\mathrm{d}}$ \\
Piperazina & 50 & $25 \pm 13,23^{\mathrm{b}}$ & $83,33 \pm 12,58^{\mathrm{c}}$ & $100 \pm 0^{\mathrm{d}}$ \\
Diluente* & & $4,33 \pm 1,73^{\mathrm{a}}$ & $9,67 \pm 5^{\mathrm{a}}$ & $22,67 \pm 10,41^{\mathrm{a}}$ \\
\hline
\end{tabular}

Médias com letras iguais, na coluna, não diferem entre si pelo teste de Student-Newman-Keuls $(\mathrm{P}>0,05)$.

* (Tween 80 a 17,5\% acrescido de DMSO a 12,5\%).

Fonte: Elaboração dos autores. 
A atividade anti-helmíntica dos extratos aquosos e etanólico do fruto da $M$. citrifolia foi avaliada no teste in vivo e seu percentual de eliminação está contido na Tabela 3. O percentual de eliminação considerando o extrato aquoso foi muito baixo, sendo inferior a $2 \%$. A eliminação utilizando o extrato etanólico da $M$. citrifolia foi semelhante ao do controle positivo (piperazina), sendo ambos inferiores a 25\%, não havendo diferença significativa $(\mathrm{P}>0,05)$. Esse baixo percentual de eliminação do H. gallinarum descrito para os extratos testados (aquoso e etanólico) e controle positivo, pode ter ocorrido devido à localização desse nematoide nas aves, uma vez que se encontram geralmente na porção final do ceco. Isso pode ter dificultado a ação do medicamento ou dos extratos no combate ao H. gallinarum. Sobral (2010), ao avaliar galinhas caipiras, testou a atividade anti-helmíntica da Operculina hamiltonii e descreveu a ineficiência dessa planta como ovicida e larvicida sobre $H$. gallinarum.

Tabela 3. Atividade anti-helmíntica dos extratos aquoso e etanólico obtidos do fruto de Morinda citrifolia na eliminação de Heterakis gallinarum em aves poedeiras $(\mathrm{n}=6)$ naturalmente infectadas.

\begin{tabular}{|c|c|c|c|}
\hline \multirow{2}{*}{ Tratamentos } & \multicolumn{2}{|c|}{ Número de helmintos } & \multirow{2}{*}{ Eliminação (\%) } \\
\hline & Exame de fezes & Necropsia & \\
\hline $\begin{array}{l}\text { Extrato Aquoso } \\
\left(50,1 \mathrm{mg} \cdot \mathrm{mL}^{-1}\right)\end{array}$ & 07 & 361 & $1,90^{\mathrm{a}}$ \\
\hline $\begin{array}{c}\text { Extrato Etanólico } \\
\left(24,6 \mathrm{mg} \cdot \mathrm{mL}^{-1}\right)\end{array}$ & 35 & 137 & $20,35^{\mathrm{b}}$ \\
\hline Água & 11 & 396 & $2,70^{\mathrm{a}}$ \\
\hline Diluente* & 06 & 113 & $5,04^{\mathrm{a}}$ \\
\hline $\begin{array}{c}\text { Piperazina } \\
\left(50 \mathrm{mg} \cdot \mathrm{mL}^{-1}\right)\end{array}$ & 18 & 69 & $20,68^{b}$ \\
\hline
\end{tabular}

Médias com letras iguais, na coluna, não diferem entre si pelo teste de Student-Newman-Keuls $(\mathrm{P}>0,05)$.

* (Tween 80 a 17,5\% acrescido de DMSO a 12,5\%).

Fonte: Elaboração dos autores.

\section{Conclusões}

No teste in vitro, as concentrações mais elevadas dos extratos aquoso e etanólico da $M$. citrifolia apresentaram elevada eficácia contra $H$. gallinarum, com percentual de mortalidade superior a $90 \%$. No entanto, uma baixa eficiência no teste in vivo foi observada, havendo necessidade de estudos com maiores concentrações.

\section{Referências}

AMATO, J. F. R.; BOEGER, W. A.; AMATO, S. B. Protocolos para laboratório - coleta e processamento de parasitos de pescado. Imprensa Universitária, UFRRJ, Rio de Janeiro. 1991. 77 p.
BOWMAN, D. D.; LYNN, R. C.; EBERRARD, M. L.; ALCARZA. Parasitologia veterinária de Georgis. 8. ed. Barueri: Manole, 2006. 422 p.

BRITO, D. R. B.; FERNANDES, R. M.; FERNANDES, M. Z. L. C. M.; FERREIRA, M. D. S.; ROLIM, F. R. L.; SILVA FILHO, M. L. Atividade anti-helmíntica dos extratos aquoso e etanólico do fruto da Morinda citrifolia sobre Ascaridia galli. Revista Brasileira de Parasitologia Veterinária, São Carlos, v. 18, n. 4, p. 32-36, 2009.

BRITO, D. R. B.; FERNANDES, R. M.; LIMA, D. C. P.; SANTOS, R. S.; FERNANDES, M. Z. L. C. M.; FERREIRA, M. D. S.; DINIZ, B. L. M. Efeito antihelmíntico da folha do noni (Morinda citrifolia L.) sobre Ascaridia galli. Ciência Animal, Fortaleza, v. 21, n. 1, p. 38-47, 2011. 
CARNEIRO, V. S. Composição e estrutura da comunidade de helmintos parasitos de galinhas, Gallus domesticus (L.), no município de Seropédica, estado do Rio de Janeiro. Tese (Doutorado em Ciências Veterinárias) - Universidade Federal Rural do Rio de Janeiro, Rio de Janeiro.

FERNANDES, M. Z. L. C. M. Estudo da atividade antihelmíntica de extratos de plantas sobre nematoides de aves Ascaridia galli (Schrank, 1788) Freeborn 1923 e Heterakis gallinarum (Schrank, 1788) Madsen, 1949. 2008. Tese (Doutorado em Parasitologia) - Universidade Federal Rural do Rio de Janeiro. Instituto de Veterinária, Seropédica.

FERNANDES, R. M. Avaliação da atividade antihelmíntica de plantas em frango de corte naturalmente infectados com Ascaridia galli (Schrank, 1788) Freeborn, 1923 e Heterakis gallinarum (Scharank, 1788) Madsen, 1949. 1998. Tese (Doutorado em Parasitologia) Universidade Federal Rural do Rio de Janeiro. Instituto de Veterinária, Seropédica.

FERNANDES, R. M.; RODRIGUES, M. L. A.; BORBA, H. R.; FERNANDES, M. Z. L. C. M.; AMORIM, A. Atividade anti-helmíntica de plantas em frangos de corte naturalmente infectados com Ascaridia galli. Arquivo Brasileiro de Medicina Veterinária e Zootecnia, Belo Horizonte, v. 57, p. 264-266, 2005. Suplemento 2.

FERNANDES, R. M.; RODRIGUES, M. L. A.; BORBA, H. R.; FERNANDES, M. Z. L. C. M.; AMORIM, A. Ausência da atividade anti-helmíntica de plantas em frangos de corte naturalmente infectados com Heterakis gallinarum (Schranck,1788) Madsen, 1949. Ciência Rural, Santa Maria, v. 34, n. 5, p. 1629-1632, 2004.

FIGUEIREDO, E. A. P.; PAIVA, D. P.; ROSA, P. S.; AVILA, V. S.; ALAMINI, D. J. D. Diferentes denominações e classificação brasileira de produção alternativa de frangos. In: CONFERÊNCIADE CIÊNCIA E TECNOlOGia AVÍCOlA, 2., Campinas. Anais... Campinas: Fundação Apinco de Ciência e Tecnologia Avícolas, 2001. p. 209-222.

FREITAS, M. G. Helmintologia veterinária. Belo Horizonte: Rabelo \& Brasil, 1977. 397 p.
KALEYSA RAJ, R. Screening of indigenous plants for anthelmintic action against human Ascaris lumbricoides: Part II. Indian Journal Physiology Pharmacology, New Delhi, v. 19, n. 1, p. 47-49, 1975.

MATOS, F. J. A. Plantas medicinais: guia de seleção e emprego de plantas usadas em fitoterapia no Nordeste do Brasil. 2. ed. Fortaleza: Imprensa Universitária/UFC, $2000.346 \mathrm{p}$.

MENEZES, R. C.; MATTOS JÚNIOR, D. G.; TORTELLY, R. M. Freqüência e patologia das infecçöes causadas por nematóides e cestóides em galinhasd'angola (Numida meleagris Linnaeus, 1758) criadas extensivamente no estado do Rio de Janeiro, Brasil. Revista Brasileira de Ciência Veterinária, Rio de Janeiro, v. 8, n. 1, p. 35-39, 2001.

PIMENTEL-GOMES, F. Curso de estatística experimental. 12. ed. Piracicaba: Nobel, 1987. 467 p.

SCHENKEL, L. C.; GOSMANN, G.; PETROVICK, P. R. Produtos de origem vegetal e o desenvolvimento de medicamentos. In: SIMÕES, C. M. O. et al. Farmacognosia: da planta ao medicamento. 3. ed. Porto Alegre/Florianópolis: Ed. da UFRGS/ Ed. da UFSC, 2001. p. 301-330.

SHILASKAR, D. V.; PARASAR, G. C. In vivo and kymographic studies on Psoralea corylifolia and piper betle against avian Ascaridia galli. Indian Veterinary Journal, Madras, v. 62, n. 5, p. 387-394, 1985.

SOBRAL, F. E. S. Eficácia anti-helmíntica da Operculina hamiltonii (g.don) D. F. Austin \& Staples (1983) e Cucurbita pepo l. sobre helmintos gastrintestinais de galinhas caipiras, Gallus domesticus. 2010. Dissertação (Mestrado em Zootecnia) - Universidade Federal de Campina Grande, Campina Grande.

STEWARD, J. S. Anthelmintic studies: I. A controlled critical entero-nemacidal test. Parasitology, v. 45, n. 3-4, p. 231-241, 1955.

UENO, H.; GONÇALVES, P. C. Manual para dignóstico das helmintoses de ruminantes. 3. ed. Tokyo: Japan International Cooperation Agency, 1994. 14 p. 